\title{
Fourier Descriptor based Isolated Marathi Handwritten Numeral Recognition
}

\author{
G. G. Rajput \\ Department of Computer Science \\ Gulbarga University \\ Gulbarga, 585106, India
}

\author{
S. M. Mali \\ Department of Computer Science \\ Solapur University \\ Solapur, 413255, India
}

\begin{abstract}
Numeral recognition remains one of the most important problems in pattern recognition. To the best of our knowledge, little work has been done in Devnagari script compared with those for non Indian scripts like Latin, Chinese and Japanese. In this paper we propose an effective method for recognition of isolated Marathi handwritten numerals written in Devnagari script. Fourier Descriptors that describe the shape of Marathi handwritten numerals are used as feature. 64 dimensional Fourier Descriptors represents the shape of numerals, invariant to rotation, scale and translation. Three different classifiers, namely, nearest neighborhood (NN), K-nearest neighborhood (KNN) and Support Vector Machine (SVM) are used independently in order to recognize test numeral. These classifiers are trained with 64 dimensional Fourier Descriptors (FD) of training samples. The proposed system is experimented with a database of 13000 samples of Marathi handwritten numerals using fivefold cross validation method for result computation. An overall recognition rate of $97.05 \%, 97.04 \%$ and $97.85 \%$ are obtained for $\mathrm{NN}, \mathrm{KNN}$ and SVM respectively.
\end{abstract}

\section{Keywords}

Handwritten numeral, Fourier Descriptors, Nearest Neighbor (NN), K-Nearest Neighbor (KNN), Support Vector Machines (SVM).

\section{INTRODUCTION}

Numeral recognition remains one of the most challenging problems in pattern recognition. This has numerous applications including those in postal sorting, bank cheque processing, and automatic data entry. Many approaches have been proposed by the researchers towards recognition of isolated handwritten numerals/characters in the literature. Research in this field has basically considered feature extraction methods, classification method, and system architectures based on different strategies, such as combination of multiple classifiers and the use of multiple features. Feature extraction methods has gained considerable attention since a discriminative feature set is considered the most important factor in achieving high recognition performance. A brief survey of feature extraction methods is reported in [1,2]. Broadly, the feature extraction methods for numerals recognition reported in the literature are based on two types of features: statistical and structural. The statistical features are derived from statistical distributions of points, such a zoning, moments, projection histograms, or direction histograms $[3,4,5]$. Structural features are based on topological and geometrical properties of the character, like strokes and their directions, chain code, image fusion, endpoints, or intersections of segments, and loops $[6,7,8]$. Further structural and statistical information are integrated to highlight different character properties, since these types of features are considered to be complementary in numeral recognition [9]. Different classifiers have been used for handwritten digit recognition, such as statistical [8] structural [10] and neural networks [11]. Recently, significant contributions towards the improvement of recognition rates have been made by means of different combination strategies [11,12,13,14], and the use of support vector machines $[15,16]$. To the best of our knowledge there are only a few research reports available on Devnagari offline handwritten character and numeral recognition. [17-26]. In this paper we propose a method for recognition of isolated Marathi handwritten numerals using Fourier Descriptors(structural feature) and the support vector machines (SVM).

The rest of the paper is organized as follows. Section 2 describes data collection and preprocessing. Feature extraction is explained in section 3. Recognition phase is presented in section 4 and experimental results are discussed in Section 5. Lastly, conclusion is given in section 6 .

\section{DATA PREPROCESSING}

Marathi, the official language of Maharashtra state, is written in Devnagari script. Devnagari is one of the most popular scripts in northern portion of India. Sanskrit, Hindi and Nepali are also written in Devnagari script. The database for handwritten Marathi numerals is created with respect to variety in handwriting style. Data collection is done on a sheet specially designed for data collection. Sample data sheet is shown in figure. 1

\begin{tabular}{|l|l|l|l|l|l|l|l|l|l|l|}
\hline 0 & 0 & 0 & 0 & 0 & 0 & 0 & 0 & 0 & 0 & 0 \\
\hline 9 & 9 & 9 & 9 & 9 & 9 & 9 & 9 & 9 & 9 & 9 \\
\hline 2 & 2 & 2 & 2 & 2 & 2 & 2 & 2 & 2 & 2 & 2 \\
\hline 3 & 3 & 3 & 3 & 3 & 3 & 3 & 3 & 3 & 3 & 3 \\
\hline
\end{tabular}

FIGURE 1: Samples of handwritten Marathi numerals 
The data sheets were scanned using a flat bed scanner at a resolution of $300 \mathrm{dpi}$ and stored as grayscale images. The raw input of the digitizer typically contains noise due to erratic hand movements and inaccuracies in digitization of the actual input. The noise present in the image is removed using median filter. Since, data is collected in a predefined format slant correction is assumed to be performed. Binarization of image is performed using Otsu's method [26]. The noise at isolated locations and spikes around the end of the numerals are removed using morphological open and close operations. A minimum bounding box is then fitted to the numeral and the numeral is cropped. To bring uniformity among the numerals the cropped numeral image is normalized to fit into a size of $40 \times 40$ pixels that is decided after taking into account different sizes. Thus, a total of 13000 binary digital images representing Marathi handwritten numerals are obtained. Each image represents a handwritten numeral (binary 1) that is unconstrained, isolated and clearly discriminated from the background (binary 0). Sample preprocessed binary images are shown in figure 2 .

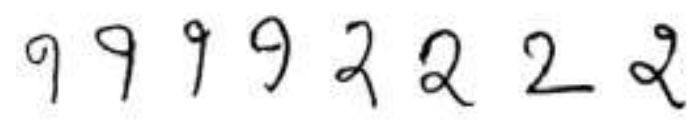

FIGURE 2: Preprocessed binary images (Numerals 1 \& 2)

\section{FEATURE EXTRACTION}

It is very important to extract the features in such a way that the recognition of numerals becomes easier on the basis of individual features of the numerals. Fourier descriptors are used to extract the features of numerals. In the proposed method, Fourier descriptors are described below.

Fourier descriptors describe the shape in terms of its spatial frequency content. Fourier descriptors are the boundary based descriptors in 2 dimensional space using the Fourier methods. As shown in figure 3, the K-point digital boundary in the $x y$-plane starting at an arbitrary point $(\mathrm{x} 0$, y0) co-ordinate pairs $(\mathrm{x} 0, \mathrm{y} 0),(\mathrm{x} 1, \mathrm{y} 1),(\mathrm{x} 2, \mathrm{y} 2), \ldots \ldots . .(\mathrm{x} \mathrm{k}-1, \mathrm{y} \mathrm{k}-1)$ are encountered in traversing the boundary say, in the counterclockwise direction.

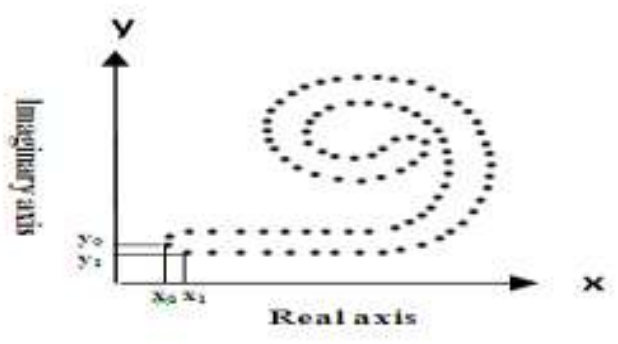

FIGURE 3: Digital boundary of an object

These co-ordinates can be expressed in the form of $x(k)=x k$ and $\mathrm{y}(\mathrm{k})=\mathrm{yk}$. With this notation, the boundary itself can be represented as sequence of co-ordinates.

$$
\mathrm{S}(\mathrm{k})=[\mathrm{x}(\mathrm{k}), \mathrm{y}(\mathrm{k})], \quad \text { for } \mathrm{k}=0,1,2, \ldots . \mathrm{K}-1 .
$$

Each co-ordinate pair can be treated as

$$
\mathrm{S}(\mathrm{k})=\mathrm{x}(\mathrm{k})+\mathrm{j} \mathrm{y}(\mathrm{k}), \quad \text { for } \mathrm{k}=0,1,2, \ldots . \mathrm{K}-1 .
$$

That is, $x$-axis is treated as real axis and the y-axis as the imaginary axis of a sequence of complex numbers.

The discrete Fourier transform (DFT) of $\mathrm{s}(\mathrm{k})$ is

$\mathrm{z}(\mathrm{u})=\frac{1}{k} \sum_{k=0}^{K-1} \mathrm{~s}(\mathrm{k}) \mathrm{e}-\mathrm{j} 2 \pi \mathrm{uk} / \mathrm{K}, \quad \mathrm{u}=0,1, \ldots, \mathrm{K}-1$

The complex coefficients a(u) are called the Fourier descriptors of the boundary. The inverse Fourier transform of these coefficients restores $\mathrm{s}(\mathrm{k})$. That is,

$$
\mathrm{s}(\mathrm{k})=\sum_{u=0}^{K-1} a(u) e^{j 2 \pi u k / K}, \quad \mathrm{k}=0,1, \ldots, \mathrm{K}-1
$$

The feature extraction process is explained below. The boundary of the preprocessed numeral image is traced in counterclockwise direction and re-sampled in order to obtain a uniform resampling along the running arc length of the boundary so that fftprocessing becomes feasible. Uniform resampling is done by assuming that the coordinates in the boundary list are the vertices of a polygon. The new boundary points are found by uniform sampling along the arc length of this polygon. The re-sampled boundary is first represented in the complex plane where the column-coordinate is the real part, and the row-coordinate the imaginary part. Thus, in this presentation, a boundary forms a contour in the complex plane (i.e. a closed curve that is uniformly sampled along the running arc length). Invariant Fourier descriptors for this boundary are computed. The invariance is obtained by -nullifying the 0-th Fourier descriptor (position invariance), dividing all Fourier descriptors by the magnitude of the 1-st Fourier descriptor (size invariance) and only considering the magnitude of the Fourier descriptors (orientation and starting point invariance).

By applying this normalization, the 0-th and 1-th Fourier descriptors do not provide any information. Hence these are eliminated. Experimentally we have chosen number of coefficients of Fourier descriptors to be 64. These 64 dimensional descriptors, invariant to scale, translation and rotation, form the feature vector. Figure 4 shows the boundary of few numerals traced.
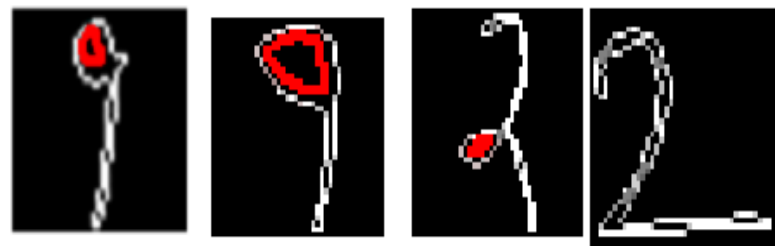

FIGURE 4: Contour of numerals 1 and 2

\section{CLASSIFICATION}

The objective of recognition is to interpret a sequence of numerals taken from the test set. Any new numeral that is to be recognized is preprocessed first. Features extracted from this numeral are sent to the Classifier. We have used three classifiers, 
namely, nearest neighbor (NN) classifier, k-nearest neighbor (K$\mathrm{NN}$ ) and SVM classifier for studying the recognition accuracy. A brief description about these classifiers is given below.

\section{Nearest neighbor classifiers}

The objective of recognition is to interpret a sequence of numerals taken from the test set. Any new numeral that is to be recognized is preprocessed first. Features extracted from this numeral are sent to the Classifier. We use nearest neighbor (NN) and k-nearest neighbor (K-NN) classifier for recognition purpose. K-Nearest Neighbor is a supervised learning algorithm. It works based on minimum distance (Euclidean Distance) from the query instance to the training samples to determine the knearest neighbor. After we gather k-nearest neighbors, we take simple majority of these k-nearest neighbors to be the prediction of the query instance. For $\mathrm{k}=1$, the classifier act as basic nearest neighbor classifier $(\mathrm{NN})$.

The 64-dimensional Fourier Descriptor that are invariant to translation, scaling and rotation are computed, as describes in Section 3 for each one of training samples and resulting set is stored as feature library. The test numerals are processed in similar manner and 64-dimensional pattern vector is computed. This pattern vector is fed to NN classifier and K-NN classifiers.

\section{Support Vector Machines}

An SVM is basically defined for two-class problem and it finds the optimal hyper-plane which maximizes the distance, the margin, between the nearest examples of both classes, named support vectors (SVs). Given a training database of $\mathrm{M}$ data: $\{\mathrm{xm} \mid$ $\mathrm{m}=1, \ldots, \mathrm{M}\}$, the linear SVM classifier is then defined as:

$$
F(x)=\sum \alpha j x j \cdot x+b
$$

where $\{x j\}$ are the set of support vectors and the parameters $\alpha j$ and $b$ have been determined by solving a quadratic problem [26].

The linear SVM can be extended to a non-linear classifier by replacing the inner product between the input vector $\mathrm{x}$ and the $\mathrm{SV}$ s $\mathrm{x}$, through a kernel function $\mathrm{K}$ defined as:

$$
\mathrm{K}(\mathrm{x}, \mathrm{y})=\phi(\mathrm{x}) \cdot \phi(\mathrm{y})
$$

This kernel function should satisfy the Mercer's Condition [27]. The performance of SVM depends on the kernel. In our study RBF (Gaussian) kernel outperformed the other commonly used kernels in the preliminary experiments.

The SVM (binary classifier) is applied to multiclass numeral recognition problem by using one-versus-rest type method. The problem now is a 10 -class problem with 10 equal to the number of segments in total. The SVM is trained with the training samples using Gaussian kernel. Fourier descriptor features of unknown numeral is computed as described in section 2 and input to the SVM for classification.

The recognition process is described in terms of algorithm below.

Input: Isolated Marathi numeral test image.

Output: Recognition of the Numeral.

\section{Method:}

i. Binarize the image to obtain numeral as 1 and background 0 .

ii. Remove noise using morphological operations.

iii. Normalize the image to a size of 40x40 pixels

iv. Trace the Boundary of the image and obtain boundary coordinates.

v. Compute 64 dimensional Fourier Descriptors from contour to obtain feature vector of size 64 .

vi. Input the test feature to classifier to recognize the test numeral.

\section{EXPERIMENTAL RESULTS}

In order to investigate the effectiveness of the proposed method, experiments were carried out on the Marathi handwritten data set obtained as described in section 2. Experiments were carried out with different number of Fourier descriptors to determine the optimal size of the feature vector. Fivefold cross validation method was used for result computation. Table provides the details of the recognition results for different size of feature vector. The results are found to be best for 64 dimensional feature vector compared to other sizes of the dimension under study, namely, 16 and 32. With 64 dimensional Fourier Descriptors overall recognition rate of $97.85 \%$ is achieved.. In result computation using five-fold technique we have used 2600 numeral images for testing and rest 10,400 images for training purpose, at each fold.

Three classifiers, namely, NN, K-NN and SVM classifiers are used independently to recognize the numerals. The proposed method was implemented using Matlab6.1 software and pattern recognition tool for Matlab (http://prtools.org/).

The performance using NN classifier in terms of the recognition rate is presented in table2. An recognition average of $97.05 \%$ is achieved. Recognition accuracy using K-NN classifier is presented in Table 3. An overall recognition of $97.04 \%$, which is approximately equal to $\mathrm{NN}$, is observed. Table 3 presents fivefold result using SVM classifier. The results are encouraging and average recognition accuracy of $97.85 \%$ is obtained.

TABLE 1: Average recognition rate using 64, 32 and 16 dimensional Fourier Descriptors respectively.

\begin{tabular}{|c|c|c|c|}
\hline Input & $\begin{array}{c}\text { Recognition } \\
\text { with 64-FD } \\
\text { in \% }\end{array}$ & $\begin{array}{c}\text { Recognition } \\
\text { with 32-FD } \\
\text { In \% }\end{array}$ & $\begin{array}{c}\text { Recognition } \\
\text { with 16-FD } \\
\text { In \% }\end{array}$ \\
\hline FOLD1 & 97.75 & 97.15 & 96.03 \\
\hline FOLD2 & 97.64 & 97.15 & 95.85 \\
\hline FOLD3 & 98.22 & 97.15 & 96.63 \\
\hline FOLD4 & 98.40 & 98.13 & 96.97 \\
\hline FOLD5 & 97.27 & 96.90 & 95.94 \\
\hline $\begin{array}{c}\text { Average } \\
\text { Recognition }\end{array}$ & 97.85 & 97.30 & 96.28 \\
\hline
\end{tabular}


TABLE 2: Recognition results using Nearest Neighborhood $(\mathrm{NN})$ classifier

\begin{tabular}{|c|c|c|c|c|c|c|}
\hline \multirow{2}{*}{$\begin{array}{c}\text { Marathi } \\
\text { Numera } \\
\text { Is }\end{array}$} & \multicolumn{5}{|c|}{ Five-Fold crossvalidation } & \multirow{2}{*}{$\begin{array}{c}\text { Average } \\
\text { Recogni } \\
\text { tion }\end{array}$} \\
\hline & 1 fold & 2 fold & 3 fold & 4 fold & 5 fold & \\
\hline 0 & 100.0 & 99.27 & 98.91 & 99.27 & 99.27 & 99.35 \\
\hline 9 & 94.18 & 96.36 & 95.64 & 95.27 & 97.09 & 95.71 \\
\hline 2 & 97.09 & 94.55 & 97.45 & 97.09 & 95.27 & 96.29 \\
\hline 2 & 96.00 & 97.45 & 94.91 & 100.0 & 97.09 & 97.09 \\
\hline 8 & 96.36 & 95.64 & 96.73 & 97.09 & 94.55 & 95.07 \\
\hline $\bar{y}$ & 98.91 & 100.0 & 95.64 & 94.91 & 99.64 & 97.82 \\
\hline$\xi$ & 93.45 & 95.64 & 96.73 & 97.82 & 93.82 & 95.49 \\
\hline$\theta$ & 98.18 & 97.09 & 96.36 & 94.18 & 93.82 & 95.93 \\
\hline$\zeta$ & 100.0 & 96.73 & 98.18 & 99.64 & 99.64 & 98.84 \\
\hline$e$ & 98.91 & 99.64 & 98.55 & 97.09 & 95.64 & 97.96 \\
\hline $\begin{array}{l}\text { Overall } \\
\text { Recog } \\
\text { nition }\end{array}$ & 97.31 & 97.24 & 96.91 & 97.24 & 96.58 & 97.05 \\
\hline
\end{tabular}

TABLE 3: Recognition results using KNN classifier

\begin{tabular}{|c|c|c|c|c|c|c|}
\hline \multirow{2}{*}{$\begin{array}{c}\text { Marathi } \\
\text { Numera } \\
\text { Is }\end{array}$} & \multicolumn{5}{|c|}{ Five-Fold crossvalidation } & \multirow{2}{*}{$\begin{array}{c}\text { Average } \\
\text { Recogni } \\
\text { tion }\end{array}$} \\
\hline & 1 fold & 2 fold & 3 fold & 4 fold & 5 fold & \\
\hline 0 & 99.64 & 99.27 & 98.91 & 99.64 & 96.36 & 98.76 \\
\hline 9 & 94.91 & 96.36 & 95.64 & 95.64 & 96.73 & 95.85 \\
\hline 2 & 97.09 & 94.55 & 97.45 & 96.73 & 94.91 & 96.15 \\
\hline 3 & 94.55 & 97.45 & 94.91 & 99.64 & 96.36 & 96.58 \\
\hline 8 & 96.73 & 95.64 & 96.73 & 97.09 & 94.55 & 96.15 \\
\hline$y$ & 97.82 & 100.0 & 95.64 & 96.73 & 98.18 & 97.67 \\
\hline$\xi$ & 94.91 & 97.45 & 96.73 & 98.18 & 94.91 & 96.44 \\
\hline$\theta$ & 97.82 & 97.09 & 96.36 & 94.55 & 94.18 & 96.00 \\
\hline$\zeta$ & 100.0 & 96.73 & 98.18 & 99.64 & 99.27 & 98.76 \\
\hline$e$ & 98.55 & 99.64 & 98.55 & 97.09 & 96.36 & 98.04 \\
\hline $\begin{array}{l}\text { Overall } \\
\text { Recog } \\
\text { nition }\end{array}$ & 97.20 & 97.42 & 96.91 & 97.49 & 96.18 & 97.04 \\
\hline
\end{tabular}

TABLE 4: Recognition results using SVM classifier

\begin{tabular}{|c|c|c|c|c|c|c|}
\hline \multirow{2}{*}{$\begin{array}{c}\text { Marathi } \\
\text { Num era } \\
\text { Is } \\
\end{array}$} & \multicolumn{5}{|c|}{ Five-Fold cross validation } & \multirow{2}{*}{$\begin{array}{c}\text { Average } \\
\text { Recogni } \\
\text { tion }\end{array}$} \\
\hline & 1 fold & 2 fold & 3 fold & 4 fold & 5 fold & \\
\hline 0 & 99.64 & 99.27 & 98.55 & 97.82 & 99.27 & 98.91 \\
\hline 9 & 93.82 & 96.73 & 98.55 & 96.36 & 99.27 & 96.95 \\
\hline 2 & 97.82 & 95.64 & 98.18 & 98.91 & 96.36 & 97.38 \\
\hline 3 & 96.36 & 97.45 & 97.09 & 99.64 & 98.55 & 97.82 \\
\hline 8 & 98.18 & 96.36 & 97.09 & 99.27 & 96.00 & 97.38 \\
\hline$y$ & 97.82 & 100.0 & 96.00 & 99.64 & 99.64 & 98.62 \\
\hline$\xi$ & 96.36 & 96.73 & 98.91 & 98.91 & 96.00 & 97.38 \\
\hline 0 & 98.91 & 97.45 & 100.0 & 94.91 & 93.45 & 96.95 \\
\hline$C$ & 100.0 & 97.09 & 98.55 & 99.64 & 99.27 & 98.91 \\
\hline$e$ & 98.55 & 99.64 & 99.27 & 98.91 & 94.91 & 98.25 \\
\hline $\begin{array}{l}\text { Overall } \\
\text { Recog } \\
\text { nition }\end{array}$ & 97.75 & 97.64 & 98.22 & 98.40 & 97.27 & 97.85 \\
\hline
\end{tabular}

The performance of the proposed method in terms of the recognition rate is compared with the contemporary work and is given in Table 5. The proposed method performs well and appears promising compared to other methods in the literature.

TABLE 5: Comparison of results of proposed method with other methods in literature

\begin{tabular}{|c|c|c|c|c|}
\hline Author & $\begin{array}{l}\text { D ata set } \\
\text { size }\end{array}$ & $\begin{array}{c}\text { Features } \\
\text { used }\end{array}$ & $\begin{array}{c}\text { Classifier } \\
\text { used }\end{array}$ & $\begin{array}{c}\text { Recognition } \\
\text { rate }\end{array}$ \\
\hline $\begin{array}{c}\text { Reena } \\
\text { B ajaj et. al. } \\
\text { [21 ] }\end{array}$ & 2460 & $\begin{array}{l}\text { Density, } \\
\text { Moment, } \\
\text { Segment }\end{array}$ & MLP & $89.68 \%$ \\
\hline $\begin{array}{c}\text { R. J. } \\
\text { Ranteke } \\
\text { et. al.[ 22 ] }\end{array}$ & 2000 & $\begin{array}{l}\text { Invariant } \\
\text { Moments }\end{array}$ & KIIII & $92.28 \%$ \\
\hline $\begin{array}{c}\text { G. G. } \\
\text { Rajput et. } \\
\text { al. [2 3] }\end{array}$ & 2000 & $\begin{array}{c}\text { Density, } \\
\text { Central } \\
\text { Moment }\end{array}$ & KIIII & $93.41 \%$ \\
\hline $\begin{array}{c}\text { M } \\
\text { H anmandlu } \\
\text { et. al. } \\
\text { [ } 17 \text { ] }\end{array}$ & 3500 & $\begin{array}{l}\text { Vector } \\
\text { distance }\end{array}$ & $\begin{array}{c}\text { Fuzzy } \\
\text { membership } \\
\text { function }\end{array}$ & $95.00 \%$ \\
\hline $\begin{array}{l}\text { U. Pal et. } \\
\text { al. [ 24] }\end{array}$ & Unknown & $\begin{array}{c}\text { Directional } \\
\text { chain } \\
\text { code }\end{array}$ & Quadratic & $98.86 \%$ \\
\hline $\begin{array}{l}\text { P. M. Patil } \\
\text { et. al. [ } 25 \text { ] }\end{array}$ & 2000 & Ring data & $\begin{array}{c}\text { Fuzzy } \\
\text { neural } \\
\text { network }\end{array}$ & $99.5 \%$ \\
\hline $\begin{array}{c}\text { Proposed } \\
\text { method }\end{array}$ & 13000 & $\begin{array}{c}\text { Fourier } \\
\text { Descriptor }\end{array}$ & S VM & $97.85 \%$ \\
\hline
\end{tabular}

\section{CONCLUSION}

In this paper we have presented a method for recognition of isolated Marathi handwritten numerals. Fourier descriptors, invariant to translation, scaling and rotation, were computed and used as features of the images representing handwritten numerals. Classification was done using NN, K-NN and multiclass SVM classifiers. Fivefold cross validation technique was used for result computation. Overall recognition rate of $97.05 \%, 97.04 \%$ and $97.85 \%$ was achieved for NN, K-NN, and SVM classifiers respectively. The proposed method is thinning free and independent of the font style written by writer. SVM classifier dominates the other classifiers in terms of better recognition rate. The main recognition errors were due to abnormal writing and ambiguity among similar shaped numerals. Future work can include improving the recognition accuracy of the individual numerals by combining the multiple classifiers.

\section{REFERENCES}

[1] Øivind Due Trier, Anil K Jain , Torfinn Taxt, Feature Extraction Methods For Character Recognition - A Survey, Pattern Recognition, vol. 29 (1996), 641-662.

[2] N. Arica and F. Yarman-Vural, "An Overview of Character Recognition Focused on Off-line Handwriting", IEEE Transactions on Systems, Man, and Cybernetics, Part C: Applications and Reviews, 31(2) (May 2001), 216 - 233.

[3] Luiz S. Oliveira, F. Bortolozzi, C.Y.Suen, "Automatic Recognition of Handwritten Numerical Strings: A Recognition and Verification Strategy", IEEE Transactions on Pattern Recognition and Machine Intelligence, Vol. 24, No. 11 (2001) 1448-1456.

[4] Heute L. T. Paquet, J. V. Moreall, A Structural/Statislical feature base vector for handwritten character recognition, Pattern Recognition Letters, 19, (1998) 629-641. 
[5] G.G. Rajput and Mallikarjun Hangarge, "Recognition of isolated handwritten Kannada numerals based on image fusion method: ",PReMI07, LNCS.4815 (2007),153-160.

[6] J. Cai, Z.Liu ,Integration of structural and statistical information for Unconstrained Handwritten Numeral Recognition. Proceedings of the Fourteen International conference on pattern Recognition, Vol. 1 (1998), .378380 .

[7] Banashree N. P., and R. Vasanta, OCR for Script Identification of Hindi (Devnagari) Numerals using Feature Sub Selection by Means of End-Point with Neuro-Memetic Model, International Journal of Intelligent Systems and Technologies 2;3(2008), 206-210.

[8] N. Sharma, U. Pal , F. Kimura and S. Pal, Recognition of Off-Line Handwritten Devnagari Characters Using Quadratic Classifier, Springer LNCS, Volume 4338(2006) 805-816.

[9] U. Pal, T. Wakabayashi and F. Kimura, " A system for offline Oriya handwritten character recognition using curvature feature ", 10th International Conference on Information Technology, IEEE (2007) 227-229.

[10] U. Bhattacharya, B.B. Chaudhari, R.Ghosh, and M.Ghosh, "On recognition of Handwritten Devnagari Numerals", In proc. Of the workshop on Learning algorithms for pattern recognition (in conjunction with the 18th Australian Joint Conference on Artificial Intelligence), Sydney (2005) 1-7.

[11] Zhang, M.Fu, H. Yan ,A Modulator Classification Scheme with Elastic Net models for handwritten digit Recognition, Vol. 31, No. 12 (1998), 1849-1864.

[12] J. Cai, Z.Liu ,Integration of structural and statistical information for Unconstrained Handwritten Numeral Recognition. Proceedings of the Fourteen International conference on Pattern Recognition, Vol. 1 (1998), 378-380.

[13] A. Majumdar and B.B. Chaudhuri, "Printed and handwritten Bangla numeral recognition using multiple classifier outputs", Proceedings of the first IEEE ICSIP06, Vol. 1 (2006) 190-195.

[14] Dinesh Achaya U, N V Subba Reddy and Krishnamoorthi, "Hierarchical Recognition System for Machine Printed Kannada Characters", IJCSNS International Journal of Computer Science and Network Security, Vol.8 No.11 ( November 2008) 44-53.

[15] L. S. Oliveira, R. Sabourin, Support Vector Machinesfor Handwritten Numerical String Recognition. 9th international workshop on Frontiers in handwriting Recognition, Kokubunji, Tokyo, Japan (Oct. 2004) 39-44.
[16] Mehmmood Abdulla Abd and George Paschos, "Effective Arabic Character Recognition using Support Vector Machines", Innovations and Advanced Techniques in Computer and Information Sciences and Engineering, Springer, (2007) 7-11.

[17] M. Hanmandlu and O. V. R. Murthy, "Fuzzy Model Based Recognition of Handwritten Hindi Numerals", In Proc. Intl. Conf. on Cognition and Recognition, (2005) 490-496.

[18] S. Kumar and C. Singh, "A Study of Zernike Moments and its use in Devnagari Handwritten Character Recognition", In Proc. Intl. Conf. on Cognition and Recognition, (2005)514-520.

[19] N. Sharma, U. Pal, F. Kimura and S. Pal, "Recognition of Offline Handwritten Devnagari Characters using Quadratic Classifier", In Proc. Indian Conference on Computer Vision Graphics and Image Processing, (2006) 805-816.

[20] U. Pal, N. Sharma, T. Wakabayashi and F. Kimura, "OffLine Handwritten Character Recognition of Devnagari Script", In Proc. 9th ICDAR, (2007) 496-500.

[21] Reena Bajaj, Lipika Dey and Santanu Chaudhur, "Devnagari numeral recognition by combining decision of multiple connectionist classifiers", Vol. 27, Part 1 (Feb. 2002) 59-72.

[22] R. J. Ramteke and S. C. Mehrotra, "Recognition of Handwritten Devnagari Numerals", International Journal of Computer Processing of Oriental Languages Ó Chinese Language Computer Society \&World Scientific Publishing Company, 2008.

[23] G. G. Rajput, S. M. Mali, Isolated Marathi Numeral Recognition using Density and Central Moment Feature, Proc. International Conference on Cognition and Recognition, (April 2008) 126-133.

[24] U. Pal, T. Wakabayashi, N. Sharma, F. Kimura, "Handwritten numeral recognition of six popular Indian scripts," Proc. 9th ICDAR, Curitiba, Brazil, Vol.2 (2007) 749-753.

[25] P.M. Patil, T.R. Sontakke, "Rotation scale and translation invariant handwritten Devanagiri numeral character recognition using fuzzy neural network", Elsevier, Pattern Recognition, vol. 40(2007) 2110-2117.

[26] G. G. Rajput, S. M. Mali, "Handwritten Marathi Numeral Recognition with Fourier Descriptors using Five Fold Cross Validation “, Computer Vision and Information Technology: Advances and Applications, I.K. Int. Pub. India (2009), 528-534.

[27] R. C. Gonzalez and R. E. Woods (2009), Digital Image Processing,3/e, Prentice Hall.

[28] Vapnik (1995), The Nature of Statistical Learning Theory, Springer Verlang. 